\title{
Rapid Downregulation of Innate Immune Cells, Interleukin-12 and Interleukin-23 in Generalized Pustular Psoriasis with Infliximab in Combination with Acitretin
}

\author{
M.M. Tang Z.Spanou H. Tang F. Schibler N.Pelivani N. Yawalkar \\ Department of Dermatology, Bern University Hospital and University of Bern, Bern, Switzerland
}

\section{Key Words}

Generalized pustular psoriasis - Tumor

necrosis factor $\cdot$ Infliximab $\cdot$ Acitretin

\begin{abstract}
Background: Generalized pustular psoriasis (GPP) is a severe inflammatory disease characterized by recurrent eruptions of sterile pustules on erythematous skin. Although tumor necrosis factor (TNF) antagonists may lead to a rapid resolution of GPP, the mechanism of action of these agents remains to be investigated. Here, we sought to evaluate markers of immune response in the skin of a patient who experienced a rapid amelioration of GPP after treatment with infliximab and acitretin. Methods: A skin biopsy was obtained before and $72 \mathrm{~h}$ after initiation of treatment. Immunohistochemical stainings were performed to characterize alterations of the infiltrates, the apoptosis marker caspase 3 and key cytokines like TNF $\alpha$, interleukin (IL)-12, IL-23 and the chemokine CXCL8/ IL-8. Results: Parallel with clinical improvement, a striking decline of neutrophils, myeloid and plasmacytoid dendritic cells, M1 macrophages and partly of CD4+ T cells was observed. There was no evidence of increased apoptosis mediated through the caspase-3 pathway. A marked reduction par-
\end{abstract}

ticularly of IL-12 and IL-23 and, to a lesser degree, of TNF $\alpha$ and CXCL8/IL-8 was observed. Conclusion: A swift clinical improvement of GPP by infliximab and acitretin is associated with a marked reduction particularly of innate and partially of the acquired immune cells as well as IL-12 and IL-23.

Copyright $\odot 2013$ S. Karger AG, Basel

\section{Introduction}

Generalized pustular psoriasis (GPP) of von Zumbusch is characterized by a sudden eruption of generalized painful pustules on an erythematous base which coalesce into lakes of pus, with high-grade fever, leukocytosis and elevated serum Creactive protein [1]. It is potentially lifethreatening and generally requires early administration of systemic anti-inflammatory agents such as oral retinoids, cyclosporine or methotrexate. The onset of action of these agents may be unsatisfactory and potentially associated with organ-specific toxicity. Previous reports indicate that tumor necrosis factor alpha (TNF $\alpha$ ) antagonists, particularly infliximab, may induce a rapid resolution of GPP [2-5]. The antiinflammatory mechanism of action of infliximab in the lesional skin of GPP re- mains to be elucidated. In this study, we investigated alterations in the cutaneous immune response in a patient with a rapid amelioration of GPP after the initiation of treatment with infliximab and acitretin.

\section{Case Report}

A 72-year old male was referred to our hospital with a 1-month history of progressive GPP which was not responding to treatment with topical corticosteroids. He had type II diabetes mellitus, hyperlipidemia, hypertension and chronic renal insufficiency. His regular medications included aspirin, atorvastatin, candesartan and amlodipine. There was no recent change of medication or clinical signs of infection. He had no previous or family history of psoriasis.

On clinical examination, there were numerous scattered pustules on an erythematous base with areas of confluence, mainly involving the trunk and extremities. Biopsy of a pustule on the right thigh was consistent with pustular psoriasis. The pustules were sterile on histological examination and culture.

Full blood count revealed a hemoglobin level of $111 \mathrm{~g} / \mathrm{l}$ (normal range 135-168 g/l)

\section{KARGER}

E-Mail karger@karger.com

www.karger.com/drm
(C) 2013 S. Karger AG, Basel

$1018-8665 / 12 / 2254-0338 \$ 38.00 / 0$ 

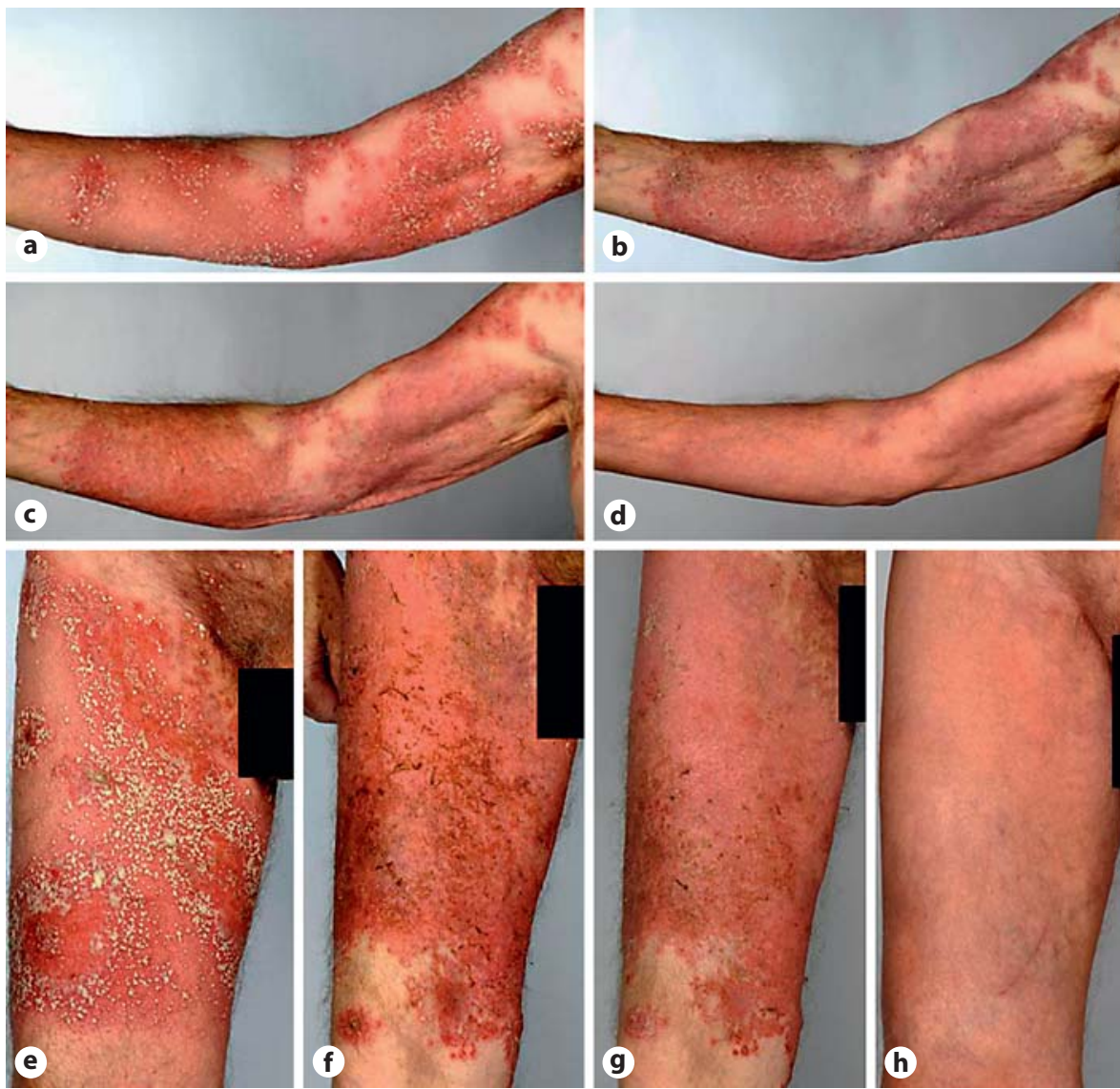

Fig. 1. a, e GPP before treatment. b, f At 24 h. c, $\mathbf{g}$ At $72 \mathrm{~h}$ after infliximab (5 mg/ $\mathrm{kg}$ ) and acitretin $35 \mathrm{mg} /$ day. d, h One month after infliximab therapy with acitretin $35 \mathrm{mg} /$ day.

and leukocytosis of $23 \times 10^{9} / 1$ (normal range 3.5-10.5) with a neutrophilia of 20.9 $\times 10^{9} / 1$ (normal range 1.6-7.4). His chest radiograph was normal and the interferon gamma (IFN $\gamma$ ) release assay was negative. Due to the severity of the disease, treatment with infliximab $(5 \mathrm{mg} / \mathrm{kg})$ in combination with acitretin ( $35 \mathrm{mg} /$ day; $0.5 \mathrm{mg} /$ $\mathrm{kg}$ body weight) was initiated. A marked improvement of pustular lesions was noted within the first $48 \mathrm{~h}$ after the first infusion with infliximab (fig. 1). Since new pustules appeared after 6 days, a second infusion of infliximab $(5 \mathrm{mg} / \mathrm{kg})$ was given and this led to a complete remission of all cutaneous lesions within the next few days. Treatment was continued for 1 month with acitretin $35 \mathrm{mg} /$ day and was subsequently tapered down over the following 4 months to 10 $\mathrm{mg} /$ day. There was a virtually complete remission for over 12 months. Thereafter, due to minor recurrent pustular eruptions on his trunk, the dosage of acitretin was not reduced further and has been maintained ever since for over 24 months.

Infliximab Abrogates Innate Immunity in Generalized Pustular Psoriasis
Punch biopsy specimens (5 $\mathrm{mm}$ ) were obtained from the lesional skin before beginning therapy and from the skin adjacent to the first biopsy after $72 \mathrm{~h}$. Tissue samples were immediately embedded in optimal cutting temperature compound, were snap-frozen and stored at $-70^{\circ} \mathrm{C}$ until sectioning. Immunohistochemistry was performed using the streptavidin-biotin complex/alkaline phosphatase method, as described previously $[6,7]$. The antibodies used as first-stage reagents included CD3 (Clone: F7.2.38, DakoCytomation, Glostrup, Denmark), CD4 (Clone: MT310, Dako), CD8 (Clone: DK25, Dako), CD69 (Clone: FN50, BD Pharmingen, N.J., USA), CD1a (Clone: 10, Dako), CD11c (Clone: KB90, Dako), CD208/DC-LAMP (Clone 104.G4, Immunotech, Prague, Czech Republic), CD303/BDCA-2 (Clone: AC144, Miltenyi Biotec, Bergisch Gladbach, Germany), CD123 (Clone: 9F5, Pharmingen), CD163 (Clone: EDHU-1, Serotec MCA, Oxford, UK), CD32 (Clone: KB61, Dako), neutrophil elastase (Clone: NP57, Dako),
HLA-DR (Clone: TAL.1B5, Dako), cleaved caspase-3 (Cell Signaling Technology, Danvers, Mass., USA), TNF $\alpha$ (R\&D Systems), interleukin (IL)-12p70 (Clone: 24945.11, R\&D Systems, AF-210-NA), IL23 (Clone: HLT2736, BioLegend, San Diego, Calif., USA) and CXCL8/IL-8 (Abcam, Cambridge, UK). Irrelevant immunoglobulin $\mathrm{G}$ subclass-matched antibodies were used for negative controls.

Quantitative analysis of positively stained cells was performed by using the digital image analysis system NIS-Elements Software BR 2.30 (Nikon, Tokyo, Japan), as previously described [7].

In order to characterize the inflammatory infiltrate, immunostaining with various cellular markers was performed before and $72 \mathrm{~h}$ after the initiation of treatment. The representative stainings and quantification of the inflammatory cells are illustrated in figures 2 and 3, respectively. In parallel with clinical improvement, a substantial reduction of the inflammatory infiltrate was seen after treatment. In par- 


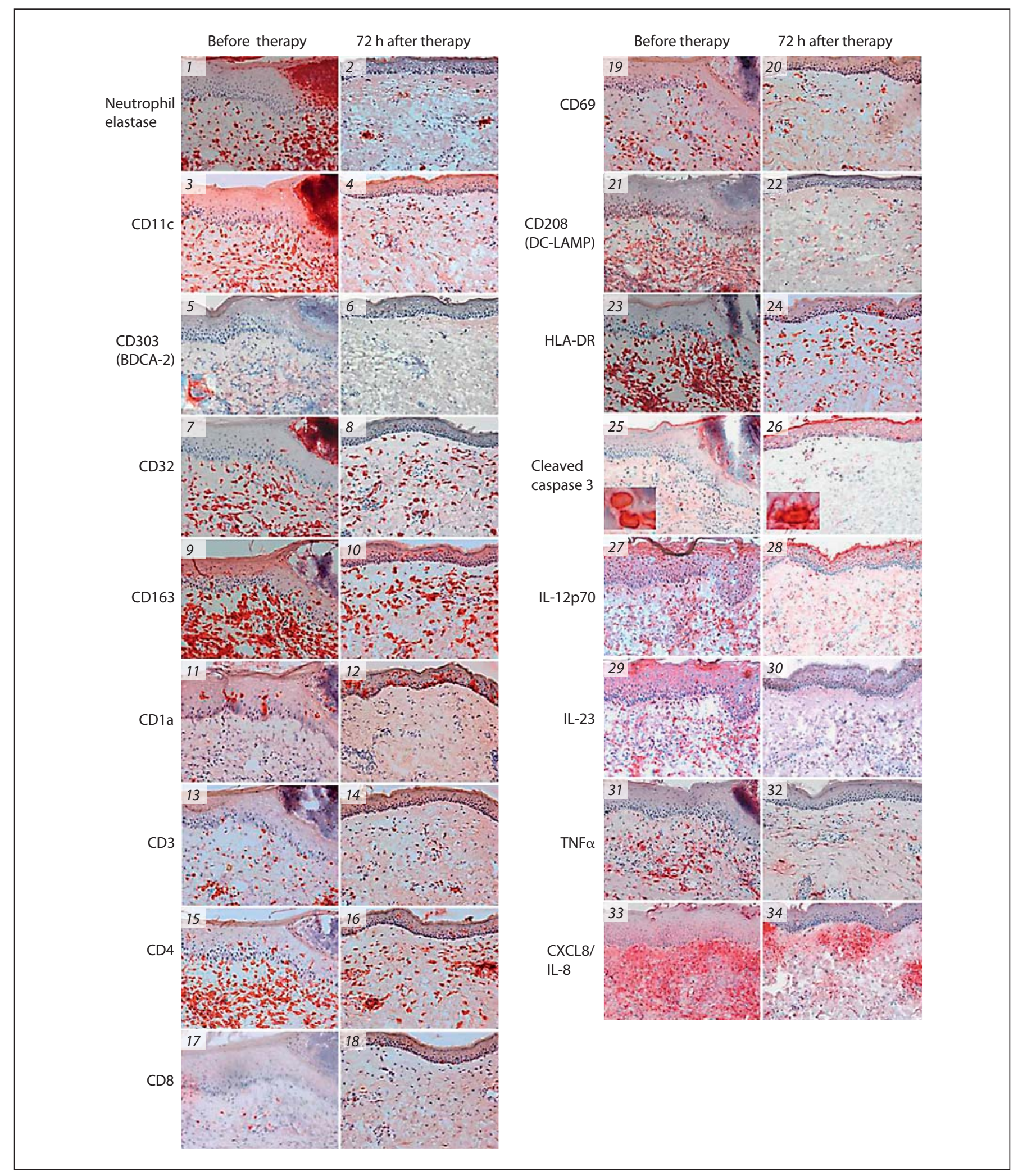

Fig. 2. Immunohistochemical analysis of different leukocyte populations, the apoptosis marker caspase 3, the cytokines TNF $\alpha$, IL-12, IL-23 and the chemokine CXCL8/IL-8 before $(1,3,5,7,9,11,13,15,17,19,21,23,25,27,29,31$ and 33) and 72 h after (2, 4, 6, 8, 10, 12, $14,16,18,20,22,24,26,28,30,32$ and 34) treatment with infliximab and acitretin $35 \mathrm{mg} /$ day. Original magnification $\times 200$. 
Fig. 3. Quantification of the inflammatory cells, cleaved caspase $3, \mathrm{TNF} \alpha, \mathrm{CXCL} 8 /$ IL-8, IL-12 and IL-23 before and $72 \mathrm{~h}$ after treatment with infliximab and acitretin $35 \mathrm{mg} /$ day. N. elastase $=$ Neutrophil elas tase.

ticular, a marked decrease of neutrophils (neutrophil elastase) and myeloid (CD11c) dendritic cells (DC) was observed. In comparison to myeloid DC (CD11c), lower numbers of plasmacytoid DC (CD303) were found scattered throughout the dermis before treatment. No positively stained CD303+ cells were observed after therapy. Although M1 and M2 macrophages (CD32 and CD163, respectively) also declined markedly, relevant remaining numbers of M2 macrophages (CD163) were still detected after treatment. A notable reduction was also observed, mainly for CD3+ and CD4+ T cells, whereas the numbers of CD8+ $\mathrm{T}$ cells declined only marginally. Before treatment, an increased expression of activation markers on T cells (CD69, HLA-DR) and DC (CD208, HLA-DR) was observed. The expression of these activation markers was also notably decreased after treatment, but was still quite evident, especially for HLA-DR. Interestingly, an increase in the number of CDla+ Langerhans cells was observed after the treatment with infliximab and acitretin.

In order to elucidate if the induction of inflammatory cell apoptosis was responsible for the rapid therapeutic response, immunohistochemical staining was performed with an anti-cleaved caspase- 3 antibody. Activated caspase-3-positive cells were identified by their bright-red appearance (fig. 2 , inset of 25,26 ) and were only occasionally detected in the epidermal and upper dermal compartment in simi-

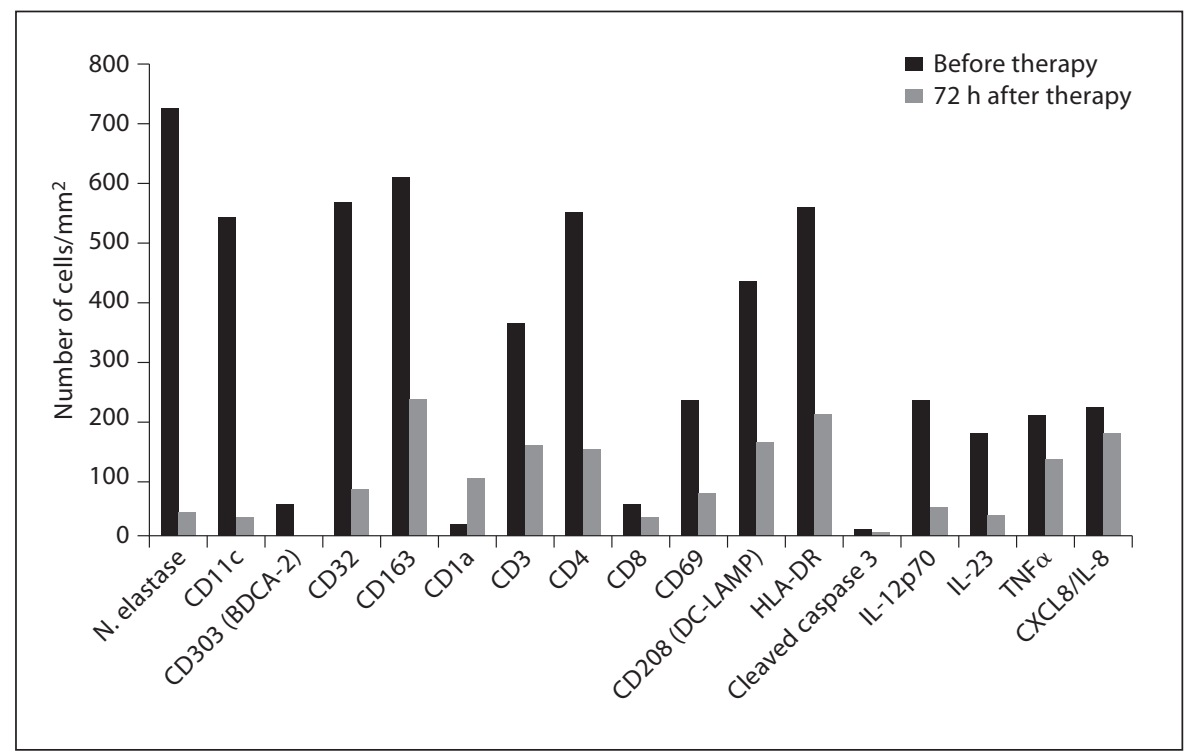

larly low numbers before and after treatment.

To further evaluate whether this treatment correlated with a downregulation of psoriasis-relevant cytokines and chemokines, immunostainings were performed for TNF $\alpha$, CXCL8/IL-8, IL-12 and IL-23. A marked decrease in the number of infiltrating cells expressing IL-12 and IL-23 was seen after therapy. A decrease was also observed for TNF $\alpha$ and CXCL8/IL-8, although substantial expression of CXCL8/ IL-8, particularly in the dermis, was still noted after treatment.

\section{Discussion}

In this study, we demonstrated that infliximab in combination with acitretin led to a rapid resolution of GPP which was subsequently maintained by low-dose acitretin. In parallel with clinical improvement a marked downregulation, particularly of the innate and partially of the acquired immune responses, was noted in the skin.

GPP is associated with significant morbidity and treatment is often challenging. There is a lack of evidenced-based therapies and universally accepted guidelines for management of this disease. Our study, together with previous reports, confirms the rapid effectiveness of infliximab on GPP within a few days [2-5]. TNF antagonists may be indicated as rescue therapy in severe GPP that is unresponsive to traditional systemic therapies. In addition, as demonstrated in this case, TNF antagonists can be helpful to achieve quick clinical control in patients with severe comorbidities which preclude the use of other systemic agents such as cyclosporine or methotrexate in high doses. In previous reports, the recurrence of pustules has been noticed between 5 and 14 days after a single dose of infliximab $[3,5]$. Similarly, in our patient, a second infusion of infliximab was required to maintain clinical remission.

The pathogenesis of GPP is still poorly understood and data on the underlying immunological mechanisms is limited. Our results show that, besides neutrophils, skin lesions in GPP are highly infiltrated with different cell populations of the innate immune system like myeloid DC, M1 and M2 macrophages. Myeloid DC and M1 macrophages represent the main source of pivotal cytokines like TNF $\alpha$, CXCL8/IL-8, IL-12 and IL-23 [8$10]$. In correlation with the increased infiltrate, an enhanced expression of these cytokines was readily found in GPP before therapy. TNF $\alpha$ has pleiotropic effects that include the upregulation of adhesion molecules (ICAM-1) which enhances leukocyte recruitment to sites of evolving inflammation and stimulation of proinflammatory cytokines and chemokines (i.e. IL-1, IL-6 and CXCL8/IL-8) [11]. Accordingly, intense immunostaining for 
CXCL8/IL-8, which plays a major part in the recruitment of neutrophils, was found in the skin lesions before therapy. Recently, an IL-36 receptor antagonist deficiency has also been reported to lead to CXCL8/IL-8 upregulation in GPP [12]. Analysis of the in situ expression of IL-36 and the IL-36 receptor antagonist during successful therapy will be of interest in future investigations.

The pathogenic role of IL-12 and IL-23, shown for the first time in GPP, has not been elucidated so far in this disease. While IL-12 stimulates Th1 cells and IFN $\gamma$ production, IL-23 leads to the activation of Th17 cells [13]. Th17 cells are characterized by their ability to produce specific cytokines such as IL-17, IL-21 and IL-22 [14]. Notably, IL-17 is involved in mediating neutrophil recruitment and activation. Thus, as in plaque psoriasis, the IL-23/IL17 pathway could also be of relevance in GPP. This is further substantiated by a recent report showing the effectiveness of ustekinumab, an IL-12/IL-23 p40 antagonist, in GPP [15].

In this study, we further investigated alterations in inflammatory cells $72 \mathrm{~h}$ after the initiation of treatment. In correlation with clinical improvement, a substantial decline in the numbers of neutrophils, activated myeloid DC and mainly M1 macrophages as well as activated CD4+ T cells was observed. A similar reduction of these cells has also been reported previously in palmoplantar pustular psoriasis after treatment with infliximab [16]. In parallel with the reduced number of these cells, a marked decrease was found for IL-12 and IL-23. Our study is also the first to demonstrate a marked decline of plasmacytoid DC which were not detectable any more in the skin lesion after therapy. Plasmacytoid DC are impor- tant sources of type 1 IFNs and these cells are thought to be crucial in the initiation of plaque psoriasis [17]. Type I IFNs can facilitate activation of myeloid DC and subsequent production of IL-12, IL-15, IL18 and IL-23 $[18,19]$. The decline of these cells may therefore also represent an important step leading to the downregulation of inflammation after therapy with infliximab.

Interestingly however, $72 \mathrm{~h}$ after the first administration of infliximab, considerable numbers of TNF $\alpha$-positive and IL8 -positive cells were still seen in the infiltrate. This may provide an explanation for the recurrence of pustules on the 6th day after the first infliximab infusion. The presence of significant remaining numbers of M2 macrophages, which may also express TNF $\alpha$, could at least partly be responsible for this observation [20].

In contrast to the decrease in the number of most cells, an increase in the number of CDla-positive Langerhans cells was observed in the epidermis along with clinical improvement. This observation has also been noticed in previous studies in psoriatic lesions after treatment with acitretin or TNF antagonists [21, 22], indicating that Langerhans cells may also have some immunomodulatory function in GPP.

The precise underlying mechanisms leading to the rapid reduction of the inflammatory infiltrate after therapy with infliximab is not completely understood. While some studies indicate that apoptosis of inflammatory cells is induced by TNF antagonists, other reports, including our data, fail to show that apoptosis mediated through the caspase- 3 pathway is involved [23-26]. The molecular mechanisms leading to the rapid reduction of various leukocyte populations in GPP after the neutral- ization of TNF remain to be delineated in future studies.

It is noteworthy that our patient was also started on acitretin $35 \mathrm{mg} /$ day together with the first infusion of infliximab. Acitretin is thought to improve psoriasis by normalizing the keratinization and proliferation of the epidermal cells as well as exerting some immunomodulatory effects like downregulating the production of proinflammatory cytokines including TNF, IL-1 and IL-6. Nevertheless, Caproni et al. [27] found that acitretin was not able to downregulate serum IL-17 and IL-22 levels after 12 weeks' treatment of plaque psoriasis in comparison to etanercept. Since acitretin has a rather slow onset of action, and as our patient only received acitretin for 3 days prior to obtaining the second biopsy specimen, it is very unlikely that the observed alterations in the inflammatory infiltrate were mediated by this drug.

In conclusion, our data support the effectiveness of infliximab in the treatment of GPP and, in particular, demonstrate the importance of the innate immune system in the manifestation of this disease.

\section{Acknowledgment}

We are indebted to U. Laederach, who provided excellent technical support. We thank Essex Chemie AG for providing infliximab for the study.

\section{Disclosure Statement}

N.Y. has served as a consultant and has received research trial support from Essex Chemie AG.

\section{References}

1 Borges-Costa J, Silva R, Gonçalves L, Filipe P, Soares de Almeida L, Marques Gomes M: Clinical and laboratory features in acute generalized pustular psoriasis: a retrospective study of 34 patients. Am J Clin Dermatol 2011;12:271-276.

2 Elewski BE: Infliximab for the treatment of severe pustular psoriasis. J Am Acad Dermatol 2002;47:796-797.
3 Schmick K, Grabbe J: Recalcitrant, generalized pustular psoriasis: rapid and lasting therapeutic response to antitumour necrosis factor-alpha antibody (infliximab). Br J Dermatol 2004;150:367.

-4 Trent JT, Kerdel FA: Tumor necrosis factor alpha inhibitors for the treatment of dermatologic diseases. Dermatol Nurs 2005; 17:97107.
5 Chandran NS, Chong WS: A dramatic response to a single dose of infliximab as rescue therapy in acute generalized pustular psoriasis of von Zumbusch associated with a neutrophilic cholangitis. Australas J Dermatol 2010;51:29-31.

6 Hassan AS, Kaelin U, Braathen LR, Yawalkar $\mathrm{N}$ : Clinical and immunopathologic findings during treatment of recalcitrant atopic eczema with efalizumab. J Am Acad Dermatol 2007;56:217-221. 
7 Schlapbach C, Ochsenbein A, Kaelin U, Hassan AS, Hunger RE, Yawalkar N: High numbers of DC-SIGN+ dendritic cells in lesional skin of cutaneous T-cell lymphoma. J Am Acad Dermatol 2010;62:995-1004.

8 Lowes MA, Chamian F, Abello MV, et al: Increase in TNF- $\alpha$ and inducible nitric oxide synthase-expressing dendritic cells in psoriasis and reduction with efalizumab (antiCD11a). Proc Natl Acad Sci USA 2005;102: 19057-19062.

-9 Benoit S, Toksoy A, Brocker EB, Gillitzer R, Goebeler M: Treatment of recalcitrant pustular psoriasis with infliximab: effective reduction of chemokine expression. Br J Dermatol 2004;150:1009-1012.

-10 Yawalkar N, Tscharner GG, Hunger RE, Hassan AS: Increased expression of IL-12p70 and IL-23 by multiple dendritic cell and macrophage subsets in plaque psoriasis. J Dermatol Sci 2009;54:99-105.

-11 Gottlieb AB, Masud S, Ramamurthi R, et al: Pharmacodynamic and pharmacokinetic response to anti-tumor necrosis factor- $\alpha$ monoclonal antibody (infliximab) treatment of moderate to severe psoriasis vulgaris. J Am Acad Dermatol 2008;48:68-75.

12 Marrakchi S, Guigue P, Renshaw BR, et al: Interleukin-36-receptor antagonist deficiency and generalized pustular psoriasis. N Engl J Med 2011;365:620-628.

13 Torti DC, Feldman SR: Interleukin-12, interleukin-23, and psoriasis: current prospects. J Am Acad Dermatol 2007;57:1059-1068.
14 Lowes MA, Kikuchi T, Fuentes-Duculan J, et al: Psoriasis vulgaris lesions contain discrete populations of Th1 and Th17 T cells. J Invest Dermatol 2008;128:1207-1211.

-15 Daudén E, Santiago-et-Sánchez-Mateos D, Sotomayor-López E, García-Díez A: Ustekinumab: effective in a patient with severe recalcitrant generalized pustular psoriasis. Br J Dermatol 2010;163:1346-1347.

16 Yawalkar N, Hunger RE: Successful Treatment of recalcitrant palmoplantar pustular psoriasis with sequential use of infliximab and adalimumab. Dermatology 2009;218: 79-83.

17 Nestle FO, Conrad C, Tun-Kyi A, et al: Plasmacytoid predendritic cells initiate psoriasis through interferon-alpha production. J Exp Med 2005;202:135-143.

18 Santini M, Lapenta C, Logozzi M, et al: Type I interferon as a powerful adjuvant for monocyte-derived dendritic cell development and activity in vitro and in Hu-PBL-SCID mice. J Exp Med 2000;191:1777-1788.

19 Paquette L, Hsu NC, Kiertscher SM, et al: Interferon-alpha and granulocyte-macrophage colony-stimulating factor differentiate peripheral blood monocytes into potent antigen-presenting cells. J Leukoc Biol 1998; 64:358-367.

20 Laskin DL: Macrophages and inflammatory mediators in chemical toxicity: a battle of forces. Chem Res Toxicol 2009;22:13761385.

21 Werner B, Bresch M, Brenner FM, Lima HC: Comparative study of histopathological and immunohistochemical findings in skin biopsies from patients with psoriasis before and after treatment with acitretin. J Cutan Pathol 2008;35:302-310.
22 Gordon KB, Bonish BK, Patel T, Leonardi CL, Nickoloff BJ: The tumour necrosis factor-alpha inhibitor adalimumab rapidly reverses the decrease in epidermal Langerhans cell density in psoriatic plaques. Br J Dermatol 2005;153:945-953.

23 Bedini C, Nasorri F, Girolomoni G, Pità O, Cavani A: Antitumour necrosis factor-alpha chimeric antibody (infliximab) inhibits activation of skin-homing $\mathrm{CD} 4+$ and $\mathrm{CD} 8+\mathrm{T}$ lymphocytes and impairs dendritic cell function. Br J Dermatol 2007;157:249-258.

24 Malaviya R, Sun Y, Tan JK, et al: Etanercept induces apoptosis of dermal dendritic cells in psoriatic plaques of responding patients. J Am Acad Dermatol 2006;55:590-597.

25 Goedkoop AY, Kraan MC, Teunissen MB, et al: Early effects of tumour necrosis factor alpha blockade on skin and synovial tissue in patients with active psoriasis and psoriatic arthritis. Ann Rheum Dis 2004;63:769-773.

26 Marble DJ, Gordon KB, Nickoloff BJ: Targeting TNFalpha rapidly reduces density of dendritic cells and macrophages in psoriatic plaques with restoration of epidermal keratinocyte differentiation. J Dermatol Sci 2007; 48:87-101.

27 Caproni M, Antiga E, Melani L, Volpi W, Del Bianco E, Fabbri P: Serum levels of IL-17 and IL-22 are reduced by etanercept, but not by acitretin, in patients with psoriasis: a randomized-controlled trial. J Clin Immunol 2009;29:210-214.
Infliximab Abrogates Innate Immunity in Generalized Pustular Psoriasis
Dermatology 2012;225:338-343 DOI: $10.1159 / 000346243$ 